\title{
THE NASH-MOSER THEOREM OF HAMILTON AND RIGIDITY OF FINITE DIMENSIONAL NILPOTENT LIE ALGEBRAS
}

\author{
ALFREDO BREGA, LEANDRO CAGLIERO, AND AUGUSTO CHAVES OCHOA
}

\begin{abstract}
We apply the Nash-Moser theorem for exact sequences of R. Hamilton to the context of deformations of Lie algebras and we discuss some aspects of the scope of this theorem in connection with the polynomial ideal associated to the variety of nilpotent Lie algebras. This allows us to introduce the space $H_{k-n i l}^{2}(\mathfrak{g}, \mathfrak{g})$, and certain subspaces of it, that provide fine information about the deformations of $\mathfrak{g}$ in the variety of $k$-step nilpotent Lie algebras.

Then we focus on degenerations and rigidity in the variety of $k$-step nilpotent Lie algebras of dimension $n$ with $n \leq 7$ and, in particular, we obtain rigid Lie algebras and rigid curves in the variety of 3-step nilpotent Lie algebras of dimension 7 . We also recover some known results and point out a possible error in a published article related to this subject.
\end{abstract}

\section{INTRODUCTION}

In this paper we will assume that all Lie algebras and representations are finite dimensional, and mostly over $\mathbb{R}$. Here we apply a finite dimensional version of the Nash-Moser theorem for exact sequences of R. Hamilton to the context of deformations in the variety of nilpotent Lie algebras. Our main results are described below.

1.1. The Nash-Moser theorem of R. Hamilton. A very well known general principle of deformation theory says that given an (algebraic) structure $\mu$, then

(1.1) $H^{2}(\mu, \mu)=0 \Rightarrow \mu$ is rigid, but the converse is not true in general.

By definition, an algebraic structure $\mu$ on a $\mathbb{K}$-vector space $V$ is rigid if the $G L(V)$-orbit of $\mu, \mathcal{O}(\mu)$, is a Zariski open set in the algebraic variety of all such algebraic structures.

Roughly speaking, when $\mathbb{K}=\mathbb{R}$ or $\mathbb{C}$, an algebraic structure $\mu$ is rigid if every small perturbation of $\mu$ is isomorphic to $\mu$. More precisely, it is known that $\mathcal{O}(\mu)$ is open in the metric topology if and only if it is open in the Zariski topology (see [NR, Proposition 17.1], see also [GK, Proposition 2]). As a consequence of this, the principle (1.1) follows from a particular instance of the Nash-Moser theorem for exact sequences of R. Hamilton as we recall below. This theorem is stated in $[\mathrm{H}]$ in terms of tame Fréchet spaces and it is related to the inverse function theorem of Nash and Moser

Partially supported by SECyT-UNC, FONCyT and CONICET grants. 
[Na, Mo]. Here, we state a finite dimensional version of the Nash-Moser theorem of R. Hamilton.

Theorem 1.1. Let $U \subset \mathbb{R}^{m}$ and $V \subset \mathbb{R}^{n}$ be open sets and let

$$
U \stackrel{F}{\rightarrow} V \stackrel{G}{\rightarrow} \mathbb{R}^{k}
$$

be a sequence of $C^{\infty}$ functions such that $G \circ F$ is constant, say $G(F(x))=0$ for all $x \in U$. Fix $a \in U$ and let $b=F(a) \in V$. If the linear sequence

$$
\mathbb{R}^{m} \stackrel{\left.d F\right|_{a}}{\longrightarrow} \mathbb{R}^{n} \stackrel{\left.d G\right|_{b}}{\longrightarrow} \mathbb{R}^{k}
$$

is exact (in the usual algebraic sense), there is an open neighborhood $W \subset$ $V \subset \mathbb{R}^{n}$ of $b$ and $a C^{\infty}$ map $H: W \rightarrow U \subset \mathbb{R}^{m}$ such that $F(H(y))=y$, for all $y \in W$ satisfying $G(y)=0$. That is

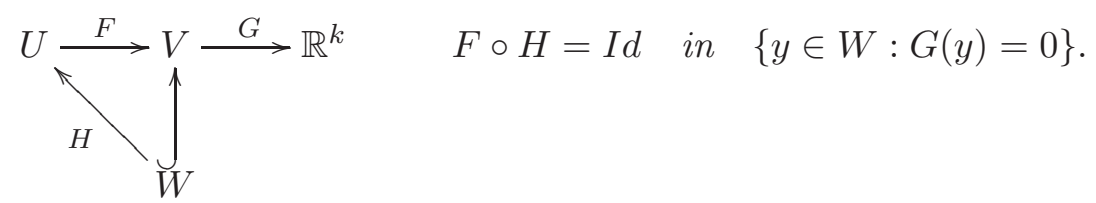

In some sense, this theorem says that the exactness of (1.3) implies a "local splitting" of (1.2).

We could not find this statement in the literature. Also, and remarkably to us, we do not see this theorem frequently cited in articles dealing with algebraic structures in the context of (1.1).

Recently, I. Struchiner pointed out to us that a close result appears in Serre's book (see in [S, pp. 89-90] the result attributed to Weil). The statement of this result is a bit weaker than Theorem 1.1. however, as the referee indicated, Serre's proof of his statement in fact proves the statement of Theorem 1.1 .

On the other hand, Hamilton's proof of his version of Theorem 1.1, in the context of tame Fréchet spaces and tame smooth functions, is considerably involved. We came across Hamilton's paper because it is cited in the survey [CSS] of M. Crainic, F. Schätz and I. Struchiner where the authors address, in a unified way, several well known problems about rigidity and stability of Lie algebras and morphisms based on the principle (1.1). To address these problems, the authors state and prove some stability results (see Propositions 4.3, 4.4 and 4.5 in [CSS]) that are phrased in terms of Kuranishi models and non-degenerate zeros of equivariant sections of vector bundles with group actions. Although the statement of Proposition 4.3 in [CSS] is more involved than that of Theorem[1.1, as in Serre's case, the proof of Proposition 4.3 of CSS provides a proof of Theorem 1.1.

We think that both, the Nash-Moser theorem of Hamilton for tame Fréchet spaces and the results about Kuranishi models and equivariant sections of CSS, are much deeper than what is needed to address some problems about rigidity and stability in a finite dimensional context.

We hope that the applications of Theorem 1.1 presented in this paper will make this classic and important result accessible to more people working on deformations of algebraic structures. 
1.2. Degenerations and rigidity of nilpotent Lie algebras. Theorem 1.1 can be applied to the study of the deformations of any algebraic structure $\mu$ on a finite dimensional $\mathbb{R}$-vector space $V$ and, in particular, when $\mu$ defines a Lie algebra structure on $V$.

Let $\mathcal{L}_{n}$ (resp. $\mathcal{N}_{n}$ ) be the algebraic variety of all Lie algebra (resp. nilpotent Lie algebra) structures $\mu$ on an $n$-dimensional vector space $\mathfrak{g}$. Let $\left\{\mathfrak{g}^{i}\right\}_{i \geq 0}$ and $\left\{\mathfrak{g}^{(i)}\right\}_{i \geq 0}$ denote, respectively, the descending central series and the derived series of $\mathfrak{g}$ (i.e. $\mathfrak{g}^{0}=\mathfrak{g}^{(0)}=\mathfrak{g}, g^{i}=\left[\mathfrak{g}^{i-1}, \mathfrak{g}\right]$ and $\left.\mathfrak{g}^{(i)}=\left[\mathfrak{g}^{(i-1)}, \mathfrak{g}^{(i-1)}\right]\right)$ and let $\mathcal{N}_{n, k}=\left\{\mathfrak{g} \in \mathcal{L}_{n}: \mathfrak{g}^{k}=0\right\}$ and $\mathcal{S}_{n, k}=\left\{\mathfrak{g} \in \mathcal{L}_{n}:\right.$ $\left.\mathfrak{g}^{(k)}=0\right\}$ be, respectively, the subvariety of all (at most) $k$-step nilpotent and solvable Lie algebras.

Theorem 1.1 applies to the study of the deformations in a given variety. For instance, if we want to study the deformations of $\mu$ in $\mathcal{L}_{n}$ we consider the following sequence of $C^{\infty}$ functions,

$$
G L(\mathfrak{g}) \stackrel{F}{\rightarrow} \Lambda^{2} \mathfrak{g}^{*} \otimes \mathfrak{g} \stackrel{G=J}{\longrightarrow} \Lambda^{3} \mathfrak{g}^{*} \otimes \mathfrak{g}
$$

where $F$ is the action of $G L(\mathfrak{g})$ on $\mu$ and $J$ is the Jacobi operator, that is

$$
\begin{aligned}
F(g)(x, y) & =g\left(\mu\left(g^{-1} x, g^{-1} y\right)\right), & & g \in G L(\mathfrak{g}) ; \\
J(\sigma)(x, y, z) & =\sum_{\text {cyclic }} \sigma(\sigma(x, y), z), & & \sigma \in \Lambda^{2} \mathfrak{g}^{*} \otimes \mathfrak{g} \quad \text { and } \quad x, y, z \in \mathfrak{g} .
\end{aligned}
$$

It turns out that the following portion of the Chevalley-Eilenberg complex for the adjoint cohomology $\mathfrak{g}$,

$$
\mathfrak{g}^{*} \otimes \mathfrak{g} \stackrel{d_{\mu}^{1}}{\longrightarrow} \Lambda^{2} \mathfrak{g}^{*} \otimes \mathfrak{g} \stackrel{d_{\mu}^{2}}{\longrightarrow} \Lambda^{3} \mathfrak{g}^{*} \otimes \mathfrak{g}
$$

is the sequence

$$
T_{I}(G L(\mathfrak{g})) \stackrel{\left.d F\right|_{I}}{\longrightarrow} T_{\mu}\left(\Lambda^{2} \mathfrak{g}^{*} \otimes \mathfrak{g}\right) \stackrel{\left.d J\right|_{\mu}}{\longrightarrow} T_{0}\left(\Lambda^{3} \mathfrak{g}^{*} \otimes \mathfrak{g}\right),
$$

(see (1.3) ) corresponding to (1.4). Therefore, if $H^{2}(\mu, \mu)=0$, we obtain from Theorem 1.1, that there exist an open neighborhood $W$ of $\mu$ in $\Lambda^{2} \mathfrak{g}^{*} \otimes \mathfrak{g}$ and a $C^{\infty}$ map $H: W \rightarrow G L(\mathfrak{g})$ such that

$$
H(\lambda) \cdot \mu=F(H(\lambda))=\lambda,
$$

for every $\lambda \in W \cap\{J=0\}=W \cap \mathcal{L}_{n}$. Hence $(\mathfrak{g}, \mu)$ is rigid in $\mathcal{L}_{n}$ (see also Theorem 5.3 of [CSS]).

On the other hand, if we are interested in the deformations of $\mu$ in $\mathcal{N}_{n, k}$ we can apply Theorem 1.1 considering the following $C^{\infty}$ functions,

$$
G L(\mathfrak{g}) \stackrel{F}{\longrightarrow} \Lambda^{2} \mathfrak{g}^{*} \otimes \mathfrak{g} \stackrel{G=J \oplus N_{k}}{\longrightarrow} \Lambda^{3} \mathfrak{g}^{*} \otimes \mathfrak{g} \oplus\left(\mathfrak{g}^{*}\right)^{\otimes(k+1)} \otimes \mathfrak{g},
$$

where $F$ is as above and $N_{k}: \Lambda^{2} \mathfrak{g}^{*} \otimes \mathfrak{g} \rightarrow\left(\mathfrak{g}^{*}\right)^{\otimes(k+1)} \otimes \mathfrak{g}$ is given by,

$$
N_{k}(\sigma)\left(x_{1}, \ldots, x_{k+1}\right)=\sigma\left(\ldots \sigma\left(\sigma\left(x_{1}, x_{2}\right), x_{3}\right), \ldots, x_{k+1}\right) \text { for } k \geq 1 .
$$

This sequence allows us to introduce the cohomology space $H_{k-n i l}^{2}(\mu, \mu)$ (see (2.3) ) obtaining that $(\mathfrak{g}, \mu)$ is rigid in $\mathcal{N}_{n, k}$ whenever $H_{k-n i l}^{2}(\mu, \mu)=0$.

Theorem 1.1 could be applied in a more subtle way. Note that $J$ and $N_{k}$ give rise, when written in coordinates, to polynomials of degree 2 and $k$ respectively. Let $I_{n, k}$ be the ideal generated by these polynomials. It turns out that, depending on $n$ and $k$, there might be polynomials of degree less 
than $k$ in the radical $\sqrt{I_{n, k}}$ that are not in $I_{n, k}$. In this case, if $P$ is such a polynomial, it can be used as (part of) the function $G$ in Theorem 1.1 to describe more precisely $\mathcal{N}_{n, k}$ which, in turn, might help to recognize rigid $\mu$ 's as points with "zero cohomology". For example, this happens for the ideal of $I_{7,6}$ (which defines $\mathcal{N}_{7}=\mathcal{N}_{7,6}$ ). Indeed, the polynomial identity $\left[\mathfrak{g}^{1}, \mathfrak{g}^{3}\right]=0$, of degree 5 , holds for every nilpotent Lie algebra $\mathfrak{g}$ of dimension 7. This is discussed in 33 and, in 4.3 , we use the identity $\left[\mathfrak{g}^{1}, \mathfrak{g}^{3}\right]=0$ and Theorem 1.1 to recover the result that states that the only three (two over $\mathbb{C}$ ) curves in $\mathcal{N}_{7}$ are rigid curves. As a byproduct we obtain a curve, consisting of solvable Lie algebras, that is rigid in the variety of Lie algebras satisfying $\left[\mathfrak{g}^{1}, \mathfrak{g}^{3}\right]=0$.

The paper also includes an analysis of all rigid Lie algebras in $\mathcal{N}_{n, k}$ for $n=5,6$. Some of these results provide examples showing that the converse part of the principle (1.1) is false in $\mathcal{N}_{5,3}, \mathcal{N}_{6,3}$, and $\mathcal{N}_{6,4}$ (in analogy with the famous example of Richardson $[\underline{\mathrm{R}}]$ in $\mathcal{L}_{18}$ ).

Finally, in 4.4, we discuss degenerations and rigidity in $\mathcal{N}_{7,3}$. As far as we know, the results of this subsection are new. We obtain three rigid Lie algebras and two (one over $\mathbb{C}$ ) rigid curves in $\mathcal{N}_{7,3}$. We also present degenerations for all Lie algebras $\mathfrak{g} \in \mathcal{N}_{7,3}$ with $\operatorname{dim} H_{3-n i l}^{2}(\mathfrak{g}, \mathfrak{g})=1$. In particular, we provide a non-trivial deformation of a Lie algebra in $\mathcal{N}_{7,3}$ which is claimed to be rigid in GR.

\section{Rigidity in the VARIETy $k$-STEP NiLPotent Lie ALGEBRAS}

Recall from the introduction that

$$
\mathcal{N}_{n, k}=\left\{\mathfrak{g} \in \mathcal{L}_{n}: \mathfrak{g}^{k}=0\right\}
$$

is the subvariety of $\mathcal{L}_{n}$ of all (at most) $k$-step nilpotent Lie algebras. Now, we will use Theorem 1.1 to discuss rigidity in $\mathcal{N}_{n, k}$.

Let $\mathfrak{g}$ be a vector space over $\mathbb{R}$ of dimension $n$. For $k \geq 1$ consider the maps

$$
N_{k} \in \operatorname{Hom}\left(\Lambda^{2} \mathfrak{g}^{*} \otimes \mathfrak{g},\left(\mathfrak{g}^{*}\right)^{\otimes(k+1)} \otimes \mathfrak{g}\right),
$$

defined inductively as follows,

$$
\begin{aligned}
& N_{1}(\mu)\left(x_{1}, x_{2}\right)=\mu\left(x_{1}, x_{2}\right), \\
& N_{k}(\mu)\left(x_{1}, \ldots, x_{k}, x_{k+1}\right)=\mu\left(N_{k-1}(\mu)\left(x_{1}, \ldots, x_{k}\right), x_{k+1}\right),
\end{aligned}
$$

where $\mu \in \Lambda^{2} \mathfrak{g}^{*} \otimes \mathfrak{g}$. It is clear that

$$
\mathcal{N}_{n, k}=\left\{\mu \in \Lambda^{2} \mathfrak{g}^{*} \otimes \mathfrak{g}: J(\mu)=0 \text { and } N_{k}(\mu)=0\right\} .
$$

It is not difficult to see that $\left.d N_{k}\right|_{\mu}: \Lambda^{2} \mathfrak{g}^{*} \otimes \mathfrak{g} \rightarrow\left(\mathfrak{g}^{*}\right)^{\otimes(k+1)} \otimes \mathfrak{g}$ is given by,

$$
\begin{aligned}
& \left.d N_{1}\right|_{\mu}(\sigma)\left(x_{1}, x_{2}\right)=\sigma\left(x_{1}, x_{2}\right), \\
& \left.d N_{2}\right|_{\mu}(\sigma)\left(x_{1}, x_{2}, x_{3}\right)=\mu\left(\sigma\left(x_{1}, x_{2}\right), x_{3}\right)+\sigma\left(\mu\left(x_{1}, x_{2}\right), x_{3}\right), \\
& \left.d N_{3}\right|_{\mu}(\sigma)\left(x_{1}, x_{2}, x_{3}, x_{4}\right)=\mu\left(\mu\left(\sigma\left(x_{1}, x_{2}\right), x_{3}\right), x_{4}\right)+\mu\left(\sigma\left(\mu\left(x_{1}, x_{2}\right), x_{3}\right), x_{4}\right) \\
& +\sigma\left(\mu\left(\mu\left(x_{1}, x_{2}\right), x_{3}\right), x_{4}\right),
\end{aligned}
$$

and so on for $k \geq 4$. 
Since $\operatorname{Im}\left(d_{\mu}^{1}\right) \subset \operatorname{Ker}\left(d_{\mu}^{2}\right) \cap \operatorname{Ker}\left(\left.d N_{k}\right|_{\mu}\right)$, in analogy with the definition of the second cohomology space $H^{2}(\mathfrak{g}, \mathfrak{g})$ we define

$$
H_{k-n i l}^{2}(\mathfrak{g}, \mathfrak{g})=\frac{\operatorname{Ker}\left(d_{\mu}^{2}\right) \cap \operatorname{Ker}\left(\left.d N_{k}\right|_{\mu}\right)}{\operatorname{Im}\left(d_{\mu}^{1}\right)}
$$

for any $k$-step nilpotent Lie algebra $(\mathfrak{g}, \mu)$. In the following theorem we apply Theorem 1.1 to obtain a rigidity result in line with the principle (1.1).

Theorem 2.1. Let $(\mathfrak{g}, \mu)$ be a k-step nilpotent Lie algebra over $\mathbb{R}$. If $H_{k-n i l}^{2}(\mathfrak{g}, \mathfrak{g})=0$, then there is a neighborhood $W$ of $\mu$ in $\Lambda^{2} \mathfrak{g}^{*} \otimes \mathfrak{g}$ and a smooth map $H: W \rightarrow G L(\mathfrak{g})$ such that $H(\lambda) \cdot \mu=\lambda$ for every $\lambda \in W \cap \mathcal{N}_{n, k}$. Hence, $(\mathfrak{g}, \mu)$ is rigid in $\mathcal{N}_{n, k}$.

Proof. Let $(\mathfrak{g}, \mu)$ be a $k$-step nilpotent Lie algebra over $\mathbb{R}$ and let $F$ and $J$ be as in (1.4). Consider the following 3 -term $C^{\infty}$-chain complex

$$
G L(\mathfrak{g}) \stackrel{F}{\longrightarrow} \Lambda^{2} \mathfrak{g}^{*} \otimes \mathfrak{g} \stackrel{G=J \oplus N_{k}}{\longrightarrow}\left(\Lambda^{3} \mathfrak{g}^{*} \otimes \mathfrak{g}\right) \oplus\left(\left(\mathfrak{g}^{*}\right)^{\otimes(k+1)} \otimes \mathfrak{g}\right) .
$$

Since $\mu$ is a $k$-step nilpotent Lie algebra we have $G(F(g))=0$ for $g \in G L(\mathfrak{g})$. Take $a=I \in G L(\mathfrak{g})$ and $b=F(I)=\mu \in \Lambda^{2} \mathfrak{g}^{*} \otimes \mathfrak{g}$ in Theorem 1.1. Since

$$
\left.d F\right|_{I}=d_{\mu}^{1} \quad \text { and }\left.\quad d G\right|_{\mu}=\left.d_{\mu}^{2} \oplus d N_{k}\right|_{\mu}
$$

it follows that the sequence

$$
\mathfrak{g}^{*} \otimes \mathfrak{g} \stackrel{\left.d F\right|_{I}}{\longrightarrow} T_{\mu}\left(\Lambda^{2} \mathfrak{g}^{*} \otimes \mathfrak{g}\right) \stackrel{\left.d G\right|_{\mu}}{\longrightarrow} T_{0}\left(\Lambda^{3} \mathfrak{g}^{*} \otimes \mathfrak{g}\right) \oplus T_{0}\left(\left(\mathfrak{g}^{*}\right)^{\otimes(k+1)} \otimes \mathfrak{g}\right),
$$

is exact if and only if $H_{k-n i l}^{2}(\mathfrak{g}, \mathfrak{g})=0$. Hence, from Theorem 1.1, it follows that if $H_{k-n i l}^{2}(\mathfrak{g}, \mathfrak{g})=0$ there exist a neighborhood $W$ of $\mu$ in $\Lambda^{2} \mathfrak{g}^{*} \otimes \mathfrak{g}$ and a $C^{\infty}$ function $H: W \rightarrow G L(\mathfrak{g})$ such that

$$
H(\lambda) \cdot \mu=F(H(\lambda))=\lambda,
$$

for every $\lambda \in W \cap\left\{J \oplus N_{k}=0\right\}=W \cap \mathcal{N}_{n, k}$ (see (2.2) ). Hence $(\mathfrak{g}, \mu)$ is rigid in $\mathcal{N}_{n, k}$, as we wanted to proof.

It is natural to ask how to extend (2.5) to an unbounded chain complex. Among other things, this would facilitate the computation of $H_{k-n i l}^{2}(\mathfrak{g}, \mathfrak{g})$ by using homological algebra tools. We have been able to do this for 2-step nilpotent Lie algebras and this work is still in progress. We point out that M. Vergne [V] introduced, as derived functors, cohomology spaces $N_{k}^{p}(\mu, \mu)$ (for $k$-step nilpotent Lie algebras) that can be viewed as certain subspaces of the regular cohomology spaces $H^{p}(\mu, \mu)$. For $p=2$, this subspace, in principle, is not quite the same as $H_{k-n i l}^{2}(\mu, \mu)$ as it consists of classes that contain a representative satisfying certain property (which depends on the representative). It would be interesting to understand better the connection between $N_{k}^{2}(\mu, \mu)$ and $H_{k-n i l}^{2}(\mu, \mu)$.

\section{The radical of the polynomial ideal Defining $\mathcal{N}_{n, k}$}

Let $\mathfrak{g}$ be a fixed $n$-dimensional vector space over $\mathbb{R}$. For $\mu \in \Lambda^{2} \mathfrak{g}^{*} \otimes \mathfrak{g}$ we know that the coordinates of $J(\mu)$ and $N_{k}(\mu)$, when expressed in terms of a basis of $\mathfrak{g}$, are, respectively, polynomials of degree 2 and $k$ in the structure constants of $\mu$, (the number of these polynomials and the number of variables 
depend on $n$ ). Let $I_{n, k}$ denote the ideal generated by these polynomials. In this subsection we will discuss briefly some algebraic properties of $I_{n, k}$ that depend on $n$. Some of these results are needed in 4.3 .

For $k \geq 3$ consider the polynomial in $\mu \in \Lambda^{2} \mathfrak{g}^{*} \otimes \mathfrak{g}$ given by,

$$
S N_{k}(\mu)\left(x_{1}, \ldots, x_{k+1}\right)=\mu\left(\mu\left(x_{1}, x_{2}\right), N_{k-2}(\mu)\left(x_{3}, \ldots, x_{k+1}\right)\right),
$$

and define

$$
\mathcal{S N}_{n, k}=\left\{\mu \in \Lambda^{2} \mathfrak{g}^{*} \otimes \mathfrak{g}: J(\mu)=0 \text { and } S N_{k}(\mu)=0\right\} \subset \mathcal{L}_{n} .
$$

It is clear that if $\mu \in \mathcal{S N}_{n, k}$ then $\mu$ defines a solvable Lie algebra. More precisely we have,

$$
\mathcal{N}_{n, k} \subset \mathcal{S N}_{n, k} \subset \mathcal{S}_{n,\left\lceil\log _{2}(k-1)\right\rceil+1} \subset \mathcal{S}_{n, k-1} \subset \mathcal{L}_{n}
$$

This follows since $\mathfrak{g}^{(i)} \subset \mathfrak{g}^{2^{i}-1}$ for $i \in \mathbb{N}$, and because $N_{k}(\mu)=J(\mu)=0$ imply $S N_{k}(\mu)=0$.

The main goal of this subsection is to point out that

$$
\begin{aligned}
\text { in general } \mathcal{N}_{n, k+1} \not \subset \mathcal{S N}_{n, k}, & & \text { for } k<n-1, \\
\text { but } \mathcal{N}_{n, k+1} \subset \mathcal{S N}_{n, k}, & & \text { for certain } k<n-1,
\end{aligned}
$$

(observe that $\mathcal{N}_{n, k}=\mathcal{N}_{n, n-1} \subset \mathcal{S N}_{n, n-1}$ for $k \geq n$ ). The inclusion (3.4) is remarkable to us since it provides "unexpected" polynomials of degree $k$ (those coming from $S N_{k}$ ) that vanish on $\mathcal{N}_{n, k+1}$. Moreover, it reveals some instances where the polynomial ideal $I_{n, k+1}$ is not radical.

More precisely, we will show next that

$$
\begin{array}{cccc}
\mathcal{N}_{5,4} \subset \mathcal{S N}_{5,3} & \mathcal{N}_{6,4} \subset \mathcal{S N}_{6,3} & \mathcal{N}_{7,4} \subset \mathcal{S N}_{7,3} & \mathcal{N}_{8,4} \not \subset \mathcal{S} \mathcal{N}_{8,3} \\
& \mathcal{N}_{6,5} \not \subset \mathcal{S N}_{6,4} & \mathcal{N}_{7,5} \not \subset \mathcal{S N}_{7,4} & \mathcal{N}_{8,5} \not \subset \mathcal{S} \mathcal{N}_{8,4} \\
& & \mathcal{N}_{7,6} \subset \mathcal{S N}_{7,5} & \text { we don't know }
\end{array}
$$

and, in general,

$$
\mathcal{N}_{2(k+1), k+1} \not \subset \mathcal{S N}_{2(k+1), k}, \quad \text { for all } k \geq 2 .
$$

We notice that, once $\mathcal{N}_{n_{0}, k+1} \not \subset \mathcal{S N}_{n_{0}, k}$ for some $n_{0}$ then $\mathcal{N}_{n, k+1} \not \subset \mathcal{S N}_{n, k}$ for all $n \geq n_{0}$. Therefore (3.5) implies that, given $k, \mathcal{N}_{n, k+1} \subset \mathcal{S N}_{n, k}$ occurs only for a finite number of $n$ 's.

3.1. Proof of $\mathcal{N}_{n, 5} \not \subset \mathcal{S N}_{n, 4}$ for $n \geq 6$. Let $\mathfrak{g}$ be the Lie algebra denoted by $12346_{E}$ in Se1 and by $\mathfrak{g}_{6,14}$ in CdGS. The structure table of $\mathfrak{g}$ is the following,

$$
\mathfrak{g}:[a, b]=c,[a, c]=d,[a, d]=e,[b, c]=e,[b, e]=f,[c, d]=-f .
$$

It is clear that $\mathfrak{g} \in \mathcal{N}_{6,5}$ (in fact $\mathfrak{g}$ is rigid in $\mathcal{N}_{6,5}$, see 4.2 ) but $\mathfrak{g} \notin \mathcal{S N}_{6,4}$, since $[[a, b],[[a, b], a]]=f \neq 0$. Therefore, $\mathcal{N}_{6,5} \not \subset \mathcal{S N}_{6,4}$ and this implies that $\mathcal{N}_{n, 5} \not \subset \mathcal{S N}_{n, 4}$ for all $n \geq 6$. 
3.2. Proof of $\mathcal{N}_{2(m+1), m+1} \not \subset \mathcal{S N}_{2(m+1), m}$ for all $m \geq 2$. Let $\mathfrak{h}_{m}$ be the Heisenberg Lie algebra $\left[x_{i}, y_{i}\right]=z(i=1, \ldots, m)$ and let $D \in \operatorname{Der}\left(\mathfrak{h}_{m}\right)$ be the derivation defined by

$$
\begin{aligned}
& D\left(x_{i}\right)=x_{i+1}, \quad i=1, \ldots, m-1 ; \\
& D\left(y_{i}\right)=-y_{i-1}, \quad i=2, \ldots, m .
\end{aligned}
$$

Then $\mathfrak{n}=\mathbb{R} D \ltimes \mathfrak{h}_{m}$ is an $(m+1)$-step nilpotent Lie algebra of dimension $2 m+2$, hence $\mathfrak{n} \in \mathcal{N}_{2 m+2, m+1}$. However, it is straightforward to verify that $\mathfrak{n} \notin \mathcal{S} \mathcal{N}_{2 m+2, m}$. In particular, this shows that

$$
\begin{array}{ll}
\mathcal{N}_{n, 4} \not \subset \mathcal{S N}_{n, 3} & \text { for } n=8 \text { and } \\
\mathcal{N}_{n, 6} \not \subset \mathcal{S N}_{n, 5} & \text { for } n=12 .
\end{array}
$$

3.3. Proof of $\mathcal{N}_{n, 4} \subset \mathcal{S N}_{n, 3}$ for $n=5,6,7$ and $\mathcal{N}_{7,6} \subset \mathcal{S N}_{7,5}$. Now we will show that

$$
\mathcal{N}_{n, 4} \subset \mathcal{S N}_{n, 3}, n=5,6,7 ; \quad \text { and } \quad \mathcal{N}_{7,6} \subset \mathcal{S N}_{7,5}
$$

One way to prove (3.6) is to use the classification of all nilpotent Lie algebras of dimension $\leq 7$ and verify (3.6) case by case. We do not know whether there is an elegant argument proving (3.6).

Another way to prove (3.6), which is more interesting to us in the context of this paper, is to show that the radical of the ideals $I_{n, 4}$ and $I_{n, 6}$ (defining $\mathcal{N}_{n, 4}$ and $\left.\mathcal{N}_{n, 6}\right)$ contain, respectively, the ideals defining $\mathcal{S N}_{n, 3}$ and $\mathcal{S N}_{n, 5}$, for the given values of $n$. We discuss this approach next.

If $\mathfrak{g}$ is a nilpotent Lie algebra of dimension $n$ with bracket given by $\mu$, then we know (by Lie's Theorem) that $\mathfrak{g}$ admits a basis $\left\{e_{i}\right\}$ such that

$$
\mu\left(e_{i}, e_{j}\right)=\sum_{k=j+1}^{n} t_{i, j, k} e_{k}, \quad \text { for } i<j .
$$

It is straightforward to see that the coordinates of $J(\mu)\left(e_{i_{1}}, e_{i_{2}}, e_{i_{3}}\right)$ and either $N_{k}(\mu)\left(e_{i_{1}}, \ldots, e_{i_{k+1}}\right)$ or $S N_{k}(\mu)\left(e_{i_{1}}, \ldots, e_{i_{k+1}}\right)$, are polynomials of degree 2 and $k$, respectively, in the variables $t_{i, j, k}$. Now we consider separately the values of $n$ given in (3.6).

Case $n=5$. Here $I_{5,4}$ is generated by

$$
P_{1}=t_{1,2,3} t_{3,4,5} \quad \text { and } \quad P_{2}=t_{1,2,4} t_{3,4,5}+t_{2,3,4} t_{1,4,5}-t_{1,3,4} t_{2,4,5},
$$

(notice that the condition $N_{4}=0$ is trivial for $n=5$ ). The condition $S N_{3}=0$ is given by $Q_{1}=Q_{2}=0$ where

$$
Q_{1}=t_{1,2,3} t_{1,3,4} t_{3,4,5} \quad \text { and } \quad Q_{2}=t_{1,2,3} t_{2,3,4} t_{3,4,5} .
$$

Then, since $P_{1}$ divides $Q_{1}$ and $Q_{2}$ we conclude that $\mathcal{N}_{5,4} \subset \mathcal{S N}_{5,3}$.

Case $n=6$. The ideal $I_{6,4}$ is generated by the polynomials below. Those having degree 2 correspond to $J$ and the one's of degree 4 correspond to $N_{4}$. 
Degree 2:

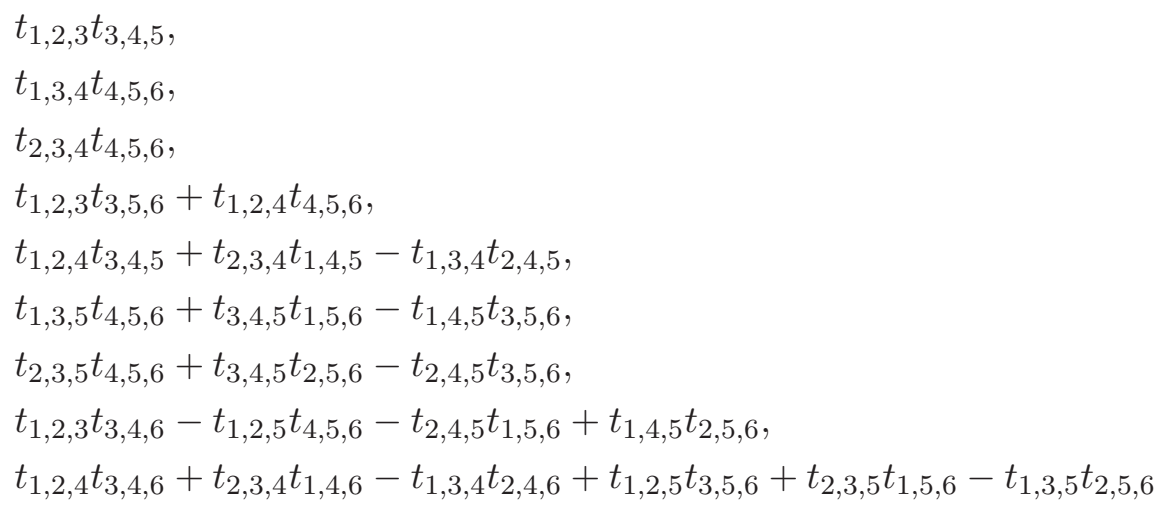

Degree 4:

$$
\begin{array}{ll}
t_{1,2,3} t_{1,3,4} t_{1,4,5} t_{1,5,6}, & t_{1,2,3} t_{2,3,4} t_{1,4,5} t_{1,5,6}, \\
t_{1,2,3} t_{1,3,4} t_{1,4,5} t_{2,5,6}, & t_{1,2,3} t_{2,3,4} t_{1,4,5} t_{2,5,6}, \\
t_{1,2,3} t_{1,3,4} t_{1,4,5} t_{3,5,6}, & t_{1,2,3} t_{2,3,4} t_{1,4,5} t_{3,5,6}, \\
t_{1,2,3} t_{1,3,4} t_{1,4,5} t_{4,5,6}, & t_{1,2,3} t_{2,3,4} t_{1,4,5} t_{4,5,6}, \\
t_{1,2,3} t_{1,3,4} t_{2,4,5} t_{1,5,6}, & t_{1,2,3} t_{2,3,4} t_{2,4,5} t_{1,5,6}, \\
t_{1,2,3} t_{1,3,4} t_{2,4,5} t_{2,5,6}, & t_{1,2,3} t_{2,3,4} t_{2,4,5} t_{2,5,6}, \\
t_{1,2,3} t_{1,3,4} t_{2,4,5} t_{3,5,6}, & t_{1,2,3} t_{2,3,4} t_{2,4,5} t_{3,5,6}, \\
t_{1,2,3} t_{1,3,4} t_{2,4,5} t_{4,5,6}, & t_{1,2,3} t_{2,3,4} t_{2,4,5} t_{4,5,6}, \\
t_{1,2,3} t_{1,3,4} t_{3,4,5} t_{1,5,6}, & t_{1,2,3} t_{2,3,4} t_{3,4,5} t_{1,5,6}, \\
t_{1,2,3} t_{1,3,4} t_{3,4,5} t_{2,5,6}, & t_{1,2,3} t_{2,3,4} t_{3,4,5} t_{2,5,6}, \\
t_{1,2,3} t_{1,3,4} t_{3,4,5} t_{3,5,6}, & t_{1,2,3} t_{2,3,4} t_{3,4,5} t_{3,5,6}, \\
t_{1,2,3} t_{1,3,4} t_{3,4,5} t_{4,5,6}, & t_{1,2,3} t_{2,3,4} t_{3,4,5} t_{4,5,6} .
\end{array}
$$

On the other hand, the condition $S N_{3}=0$ is $\left\{Q_{i}=0: i=1, \ldots, 14\right\}$ where the $Q_{i}$ 's are the following polynomials of degree 3 :

$$
\begin{array}{ll}
Q_{1}=t_{1,2,3} t_{1,3,4} t_{3,4,5}, & Q_{2}=t_{1,2,3} t_{2,3,4} t_{3,4,5}, \\
Q_{3}=t_{1,3,4} t_{1,4,5} t_{4,5,6}, & Q_{4}=t_{1,3,4} t_{2,4,5} t_{4,5,6}, \\
Q_{5}=t_{1,3,4} t_{3,4,5} t_{4,5,6}, & Q_{6}=t_{1,4,5} t_{2,3,4} t_{4,5,6}, \\
Q_{7}=t_{2,3,4} t_{2,4,5} t_{4,5,6}, & Q_{8}=t_{2,3,4} t_{3,4,5} t_{4,5,6}, \\
Q_{9}=\left(t_{1,3,4} t_{2,3,5}-t_{1,3,5} t_{2,3,4}\right) t_{4,5,6}, & \\
Q_{10}=t_{1,2,3} t_{1,4,5} t_{3,5,6}+t_{1,2,4} t_{1,4,5} t_{4,5,6}, & \\
Q_{11}=t_{1,2,3} t_{2,4,5} t_{3,5,6}+t_{1,2,4} t_{2,4,5} t_{4,5,6}, & \\
Q_{12}=t_{1,2,3} t_{3,4,5} t_{3,5,6}+t_{1,2,4} t_{3,4,5} t_{4,5,6}, & \\
Q_{13}=t_{1,2,3} t_{1,3,4} t_{3,4,6}+t_{1,2,3} t_{1,3,5} t_{3,5,6}+t_{1,2,4} t_{1,3,5} t_{4,5,6}-t_{1,2,5} t_{1,3,4} t_{4,5,6}, \\
Q_{14}=t_{1,2,3} t_{2,3,4} t_{3,4,6}+t_{1,2,3} t_{2,3,5} t_{3,5,6}+t_{1,2,4} t_{2,3,5} t_{4,5,6}-t_{1,2,5} t_{2,3,4} t_{4,5,6}
\end{array}
$$

It turns out that $Q_{i} \in I_{6,4}$ for $i=1, \ldots, 12$, that $Q_{13}, Q_{14} \notin I_{6,4}$ but $Q_{13}^{2}, Q_{14}^{2} \in I_{6,4}$. This shows that $\mathcal{N}_{6,4} \subset \mathcal{S N}_{6,3}$. 
What it is surprising to us is that $Q_{13}, Q_{14} \notin I_{6,4}$. This shows that $I_{6,4}$ is not a radical ideal. To prove this we assume in all the polynomials that,

$$
t_{1,2,4}=t_{1,3,4}=t_{1,4,5}=t_{1,4,6}=t_{2,3,5}=t_{2,5,6}=t_{3,4,5}=t_{3,5,6}=t_{4,5,6}=0 .
$$

Under this assumption, $I_{6,4}$ is generated by the polynomials,

$$
t_{1,2,3} t_{2,3,4} t_{2,4,5} t_{1,5,6} \quad \text { and } \quad t_{1,2,3} t_{3,4,6}-t_{2,4,5} t_{1,5,6},
$$

while $Q_{14}$ becomes

$$
t_{1,2,3} t_{2,3,4} t_{3,4,6} \text {. }
$$

A straightforward computation shows that $Q_{14}$ does not belong to the ideal generated by the polynomials in (3.7). If instead of assuming $t_{1,3,4}=0$ we assume that $t_{2,3,4}=0$ we obtain that $Q_{13} \notin I_{6,4}$.

Case $n=7$. In analogy to the previous cases, we have verified with the software Singular that:

- the square of the ideal defining $\mathcal{S N}_{7,3}$ is contained in $I_{7,4}$,

- the square of the ideal defining $\mathcal{S N}_{7,5}$ is contained in $I_{7,6}$,

- there are polynomials defining $\mathcal{S N}_{7,3}$ not belonging to $I_{7,4}$, as well as polynomials defining $\mathcal{S N}_{7,5}$ not belonging to $I_{7,6}$.

Again, $I_{7,4}$ and $I_{7,6}$ are not radical ideals for $n=7$.

This shows that, for $n \leq 7, S N_{3}(\mu)$ produces polynomials of degree 3 in the radical of the ideal generated by $J(\mu)$ and $N_{4}(\mu)$; and $S N_{5}(\mu)$ produces polynomials of degree 5 in the radical of the ideal generated by $J(\mu)$ and $N_{6}(\mu)$. This give rise to the following question:

Questions. Given $n>k$ :

- Is the ideal $I_{n, k}$ radical?

- Are there polynomials of degree less than $k$ in $\sqrt{I_{n, k}}$ which are not in $I_{n, k}$ ?

- Does $S N_{k-1}$ vanishes on $\mathcal{N}_{n, k}$ ? Or more generally, is there any other polynomial identity of degree less that $k$ (different from Jacobi) vanishing on $\mathcal{N}_{n, k}$ ?

For example, in this section we have shown that

- $S N_{3}$ vanishes in $\mathcal{N}_{n, 4}$ for $n=5,6,7$ but does not for $n=8$;

- $S N_{5}$ vanishes in $\mathcal{N}_{7,6}$ but does not vanishes in $\mathcal{N}_{12,6}$.

Above questions are motivated by the idea of obtaining a polynomial $P(\mu)$, simpler than $J(\mu) \oplus N_{k}$, to be used as (part of) the function $G$ in Theorem 1.1 (or more precisely, in the display (2.4) of Theorem 2.1) to study rigidity in $\mathcal{N}_{n, k}$. This could help to recognize more easily rigid Lie algebras in $\mathcal{N}_{n, k}$. This tool is used in 44.3 to find rigid curves in $\mathcal{N}_{7, k}$, for $k=3,6$.

\section{Deformations And Rigidity in $\mathcal{N}_{n, k}$, FOR $n=5,6,7$}

In this section we will consider several structure tables of Lie algebras. In order to shorten the description of these tables we will denote by $a b$ the Lie bracket $[a, b]$. 
4.1. A rigid Lie algebra with $H_{k-n i l}^{2}(\mathfrak{g}, \mathfrak{g}) \neq 0$. It is well known that a Lie algebra $\mathfrak{g}$ may be rigid but fail to satisfy $H^{2}(\mathfrak{g}, \mathfrak{g})=0$; the examples are in general involved, see for instance $[\mathbb{R}$. In this subsection we present a Lie algebra $\mathfrak{g}$ that is rigid in the variety $\mathcal{N}_{5,3}$ but $H_{3-\text { nil }}^{2}(\mathfrak{g}, \mathfrak{g}) \neq 0$.

There are only eight non-abelian Lie algebras of dimension 5 over the real numbers $\mathrm{dG}$. If $\mathfrak{f}_{n}$ denotes the standard filiform Lie algebra of dimension $n$, these Lie algebras are

\begin{tabular}{llc}
$k$ & $k$-step Lie algebra $\mathfrak{g}$ & $\operatorname{dim} H_{k-n i l}^{2}(\mathfrak{g}, \mathfrak{g})$ \\
\hline 2 & $\mathfrak{f}_{3} \oplus \mathbb{R}^{2}$ & $11=20-9$ \\
& $\mathfrak{g}_{5,1}: a b=e, c d=e$. & $0=10-10$ \\
& $\mathfrak{g}_{5,2}: a b=d, a c=e$. & $0=12-12$ \\
3 & $\mathfrak{f}_{4} \oplus \mathbb{R}$ & $4=18-14$ \\
& $\mathfrak{g}_{5,3}: a b=d, a d=e, b c=e$. & $2=17-15$ \\
& $\mathfrak{g}_{5,4}: a b=c, a c=d, b c=e$. & $0=15-15$ \\
4 & $\mathfrak{f}_{5}$ & $1=17-16$ \\
& $\mathfrak{g}_{5,6}: a b=c, a c=d, a d=e, b c=e$. & $0=17-17$
\end{tabular}

The list of 5-dimensional non-abelian nilpotent Lie algebras

and the Hasse diagram is (see [GO]),

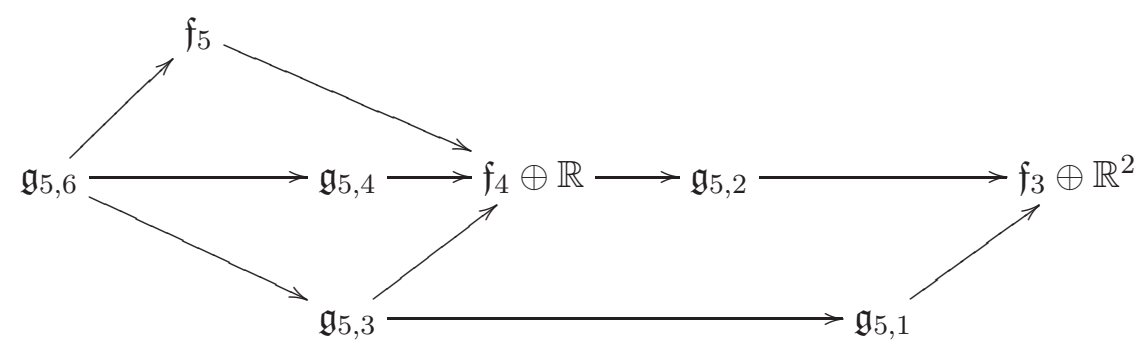

This shows that $\mathfrak{g}_{5,3}$ is rigid in the variety of 3 -step nilpotent Lie algebras but it tuns out that $H_{3-n i l}^{2}\left(\mathfrak{g}_{5,3}, \mathfrak{g}_{5,3}\right)=\operatorname{span}\left\{\nu_{1}, \nu_{2}\right\}$ with

$$
\begin{aligned}
& \nu_{1}(b, c)=c, \\
& \nu_{2}(a, b)=b, \quad \nu_{2}(a, c)=-c, \quad \nu_{2}(a, d)=-d .
\end{aligned}
$$

In fact, if $\mu$ is the bracket of $\mathfrak{g}_{5,3}$, then $\mu+t \nu_{1}$ and $\mu+t \nu_{2}$ are solvable deformations of $\mathfrak{g}_{5,3}$.

4.2. Rigid nilpotent Lie algebras in $\mathcal{N}_{6, k}$. The Hasse diagram of the 6 -dimensional nilpotent Lie algebras is given in [Se1. There are 34 real 6-dimensional nilpotent Lie algebras CdGS and being this a finite number it follows, as in dimension 5, that a Lie algebra is rigid in its class if and only if it is not a degeneration of any other in its class. It follows from the Hasse diagram in Se1 that there are: one rigid Lie algebra in $\mathcal{N}_{6,5}$, three (two over $\mathbb{C}$ ) rigid Lie algebras in $\mathcal{N}_{6,4}$, four (two over $\mathbb{C}$ ) in $\mathcal{N}_{6,3}$ and three (two over $\mathbb{C}$ ) in $\mathcal{N}_{6,2}$. The following table summarizes this information. 


\begin{tabular}{lllc} 
& $k$-step Lie algebra & $k$-step Lie algebra & \\
$k$ & $\mathfrak{g}$ as denoted in [Se1] & $\mathfrak{g}$ as denoted in [CdGS] & $\operatorname{dim} H_{k-\text { nil }}^{2}(\mathfrak{g}, \mathfrak{g})$ \\
\hline 2 & 36 & $\mathfrak{g}_{6,26}$ & $0=18-18$ \\
& $13+13$ & $\mathfrak{g}_{6,22}, t=-1,1$ & $0=20-20$ \\
3 & $246_{E}$ & $\mathfrak{g}_{6,24}, t=-1,1$ & $2=26-24$ \\
& $136_{A}$ & $\mathfrak{g}_{6,19}, t=-1,1$ & $0=25-25$ \\
4 & 1246 & $\mathfrak{g}_{6,13}$, & $1=27-26$ \\
& $1346_{C}$ & $\mathfrak{g}_{6,21}, t=-1,1$ & $0=26-26$ \\
5 & $12346_{E}$ & $\mathfrak{g}_{6,14}$ & $0=28-28$
\end{tabular}

The list of rigid 6-dimensional nilpotent Lie algebras in $\mathcal{N}_{6, k}$

In this case there are three rigid nilpotent Lie algebras with non-zero cohomology $H_{k-n i l}^{2}(\mathfrak{g}, \mathfrak{g})$, these are $\mathfrak{g}_{6,13}$ and $\mathfrak{g}_{6,24}, t=-1,1$; and the nonzero cohomology classes correspond to infinitesimal solvable deformations.

4.3. The two rigid curves in $\mathcal{N}_{7}$. It is known that there are no rigid Lie algebras in $\mathcal{N}_{7}$ (see $[\mathrm{Ca}$ ) and that there are only three (two over $\mathbb{C}$ ) rigid curves of non-isomorphic Lie algebras in $\mathcal{N}_{7}$ (see [GA]).

One of these curves consists of 6-step nilpotent Lie algebras and it is denoted as $\mathfrak{g}_{I}(\alpha)$ by Burde $\left[\mathrm{Bu}\right.$, as $\mathfrak{g}_{7,1.1}\left(i i_{\lambda}\right)$ by Magnin [Ma] and as $123457_{I}$ by Seeley [Se2]. If $\mathfrak{g}_{6}(r, t)$ is the surface of (solvable) Lie algebras given by the following structure table

$$
\begin{array}{r}
\mathfrak{g}_{6}(r, t): a b=c, a c=d, a d=e, a e=f, a f=g, a g=r g, \\
b c=e, b d=f, b e=r t f+(1-t) g, b f=r g, b g=r^{2} g, \\
c d=-r t f+t g,
\end{array}
$$

then $\mathfrak{g}_{6}(0, \alpha)$ is exactly $\mathfrak{g}_{I}(\alpha)$.

The other two curves consist of 5-step nilpotent Lie algebras and they coincide over $\mathbb{C}$. Over the complex numbers, this curve is denoted as $\mathfrak{g}_{1}(\lambda)$ by Burde [Bu], as $\mathfrak{g}_{7,0.4}(\lambda)$ by Magnin [Ma] and as $12457_{N}$ by Seeley [Se2]. The structure table of $\mathfrak{g}_{1}(\lambda)$ is obtained by setting $(r, t)=(0, \lambda)$ in the following surface of solvable Lie algebras,

$$
\begin{array}{r}
\mathfrak{g}_{5}(r, t): a b=(1+t r) c, a c=d, a d=f+t g, a e=g, a f=-r f+g, \\
b c=e, b d=g, b e=r d+f, c e=g .
\end{array}
$$

It is easy to check that both surfaces, $\left\{\mathfrak{g}_{5}(r, t)\right\}_{(r, t) \in \mathbb{R}^{2}}$ and $\left\{\mathfrak{g}_{6}(r, t)\right\}_{(r, t) \in \mathbb{R}^{2}}$, are contained in $\mathcal{S N}_{7,5}$ (see 93 for the definition). In addition, a Lie algebra in either of these curves is nilpotent if and only if $r=0$.

Therefore if $\mu(r, t)$ is the Lie algebra structure of either $\mathfrak{g}_{5}(r, t)$ or $\mathfrak{g}_{6}(r, t)$ we consider the following 3 -term $C^{\infty}$-chain complex

$$
\mathbb{R}^{2} \times G L(\mathfrak{g}) \stackrel{F}{\longrightarrow} \Lambda^{2} \mathfrak{g}^{*} \otimes \mathfrak{g} \stackrel{G=J \oplus S N_{5}}{\longrightarrow}\left(\Lambda^{3} \mathfrak{g}^{*} \otimes \mathfrak{g}\right) \oplus\left(\left(\mathfrak{g}^{*}\right)^{\otimes 6} \otimes \mathfrak{g}\right),
$$

where $S N_{5}$ is as in (3.1) and $F(r, t, g)=g \cdot \mu(r, t)$. 
The corresponding linear chain complex of the tangent spaces at the points $\left(r_{0}, t_{0}, I\right) \in \mathbb{R}^{2} \times G L(\mathfrak{g})$ and $\mu\left(r_{0}, t_{0}\right) \in \Lambda^{2} \mathfrak{g}^{*} \otimes \mathfrak{g}$ is

$$
\mathbb{R}^{2} \times \mathfrak{g}^{*} \otimes \mathfrak{g} \stackrel{\left.d F\right|_{\left(r_{0}, t_{0}, I\right)} ^{\longrightarrow}}{\longrightarrow} \Lambda^{2} \mathfrak{g}^{*} \otimes \mathfrak{g} \stackrel{\left.d G\right|_{\mu\left(r_{0}, t_{0}\right)}}{\longrightarrow}\left(\Lambda^{3} \mathfrak{g}^{*} \otimes \mathfrak{g}\right) \oplus\left(\left(\mathfrak{g}^{*}\right)^{\otimes 6} \otimes \mathfrak{g}\right)
$$

where

$$
\begin{aligned}
\left.d F\right|_{\left(r_{0}, t_{0}, I\right)} & =\left[\left.\partial_{r}\right|_{\left(r_{0}, t_{0}\right)} \mu,\left.\partial_{t}\right|_{\left(r_{0}, t_{0}\right)} \mu, d_{\mu\left(r_{0}, t_{0}\right)}^{1}\right], \\
\left.d G\right|_{\mu\left(r_{0}, t_{0}\right)} & =\left[\begin{array}{c}
d_{\mu\left(r_{0}, t_{0}\right)}^{2} \\
\left.d\left(S N_{5}\right)\right|_{\mu\left(r_{0}, t_{0}\right)}
\end{array}\right] .
\end{aligned}
$$

A computer calculation shows that the chain complex (4.1) is exact for all $\left(r_{0}, t_{0}\right)$ with $r_{0} t_{0} \neq-1$, if $\mu\left(r_{0}, t_{0}\right)$ is the structure of $\mathfrak{g}_{5}\left(r_{0}, t_{0}\right)$; and for all $\left(r_{0}, t_{0}\right)$ with $t_{0} \neq 0$, if $\mu\left(r_{0}, t_{0}\right)$ is the structure of $\mathfrak{g}_{6}\left(r_{0}, t_{0}\right)$.

The sizes of $\left.d F\right|_{\left(r_{0}, t_{0}, I\right)}$ and $\left.d G\right|_{\mu\left(r_{0}, t_{0}\right)}$ are, respectively, $147 \times 51$ and $7^{7} \times 147$. Writing down a computer code to obtain these matrices and confirm the above claims is not such a difficult task. Doing this by hand would be a huge effort, yet not impossible. For this, it could be convenient to use explicit ordinary cohomology classes in $H^{2}\left(\mu\left(r_{0}, t_{0}\right), \mu\left(r_{0}, t_{0}\right)\right)$ which can be found in [Ma] (if $\mu\left(r_{0}, t_{0}\right)$ is generic in either curve, then $\operatorname{dim} H^{2}\left(\mu\left(r_{0}, t_{0}\right), \mu\left(r_{0}, t_{0}\right)\right)=$ $9)$. Then one should show that $\left.d\left(S N_{5}\right)\right|_{\mu\left(r_{0}, t_{0}\right)}$ has no non-trivial kernel within the space generated by these clases and the two tangent vectors corresponding to $\mathbb{R}^{2}$.

Next we use the exactness of (4.1) to obtain the following proposition.

Proposition 4.1. The curves $\left\{\mathfrak{g}_{1}(\lambda): \lambda \in \mathbb{R}\right\}$ and $\left\{\mathfrak{g}_{I}(\alpha): \alpha \in \mathbb{R}, \alpha \neq 0\right\}$ are rigid curves in $\mathcal{N}_{7}$. Moreover, for any $r_{0} \neq 0$, the curves $\left\{\mathfrak{g}_{5}\left(r_{0}, t\right): t \in\right.$ $\left.\mathbb{R}, t \neq-1 / r_{0}\right\}$ and $\left\{\mathfrak{g}_{6}\left(r_{0}, t\right): t \in \mathbb{R}, t \neq 0\right\}$ are rigid curves in $\mathcal{S N}_{7,5}$.

Proof. Recall that $\mathfrak{g}_{6}(0, t) \simeq \mathfrak{g}_{I}(t)$ and $\mathfrak{g}_{5}(0, t) \simeq \mathfrak{g}_{1}(t)$. Fix $t_{0} \in \mathbb{R}$ (and $t_{0} \neq 0$ if $\mu\left(0, t_{0}\right)$ is the structure of $\left.\mathfrak{g}_{6}\left(0, t_{0}\right)\right)$. It follows from Theorem 1.1 and the exactness of (4.1) that there is a neighborhood $U \subset \mathcal{S N}_{7,5}$ of $\mu\left(0, t_{0}\right)$ such that for any Lie algebra structure $\nu \in U$ there exists $(r, t) \in \mathbb{R}^{2}$ such that $\nu \simeq \mu(r, t)$. If in addition $\nu$ is nilpotent (that is $\nu \in U \cap \mathcal{N}_{7}$ ), then $r$ must be 0 as $\mu(r, t)$ is nilpotent if and only if $r=0$. This proves that $\left\{\mathfrak{g}_{I}(\alpha): \alpha \in \mathbb{R}, \alpha \neq 0\right\}$ and $\left\{\mathfrak{g}_{1}(\lambda): \lambda \in \mathbb{R}\right\}$ are rigid curves in $\mathcal{N}_{7}$.

Now fix $r_{0} \neq 0$, and $t_{0} \neq-1 / r_{0}$ if $\mu\left(r_{0}, t_{0}\right)$ is the structure of $\mathfrak{g}_{5}\left(r_{0}, t_{0}\right)$; or $t_{0} \neq 0$ if $\mu\left(r_{0}, t_{0}\right)$ is the structure of $\mathfrak{g}_{6}\left(r_{0}, t_{0}\right)$. It tuns out that a computer calculation shows that (4.1) is still exact if we consider the function $F$ (and its differential) with the variable $r$ fixed at $r=r_{0}$. Now Theorem 1.11implies that there is a neighborhood $U \subset \mathcal{S N}_{7,5}$ of $\mu\left(r_{0}, t_{0}\right)$ such that for any Lie algebra structure $\nu \in U$ there exists $t \in \mathbb{R}$ such that $\nu \simeq \mu\left(r_{0}, t\right)$. This proves that $\left\{\mathfrak{g}_{5}\left(r_{0}, t\right): t \in \mathbb{R}, t \neq-1 / r_{0}\right\}$ and $\left\{\mathfrak{g}_{6}\left(r_{0}, t\right): t \in \mathbb{R}, t \neq 0\right\}$ are rigid curves in $\mathcal{S N}_{7,5}$.

Remark 4.2. We point out that even when $r_{1} \neq r_{2}$, the orbit of the curve $\left\{\mu\left(r_{1}, t\right): t \in \mathbb{R}\right\}$ might have non-empty intersection with the orbit of $\left\{\mu\left(r_{2}, t\right): t \in \mathbb{R}\right\}$. Therefore, even though the exactness of (4.1) implies that the set $\{\mu(r, t): r, t \in \mathbb{R}\}$ is rigid in $\mathcal{S N}_{7,5}$, this set does not constitute a rigid surface of pairwise non-isomorphic Lie algebras in $\mathcal{S N}_{7,5}$. 
4.4. Deformations and rigidity in $\mathcal{N}_{7,3}$. The goal of this subsection is to obtain all rigid points and curves in $\mathcal{N}_{7,3}$. According to the classification, there are more than fifty isomorphism classes and two 1-parameter curves. Specifically, in this subsection we do the following:

(a) With the assistance of a computer, we obtain all Lie algebras $\mathfrak{g} \in \mathcal{N}_{7,3}$ such that $H_{3-\text { nil }}^{2}(\mathfrak{g}, \mathfrak{g})=0$ (there are four such Lie algebras). According to Theorem 2.1 these Lie algebras are rigid in $\mathcal{N}_{7,3}$.

(b) Similarly, we obtain that $\operatorname{dim} H_{3-n i l}^{2}(\mathfrak{g}, \mathfrak{g})=1$ for $\mathfrak{g}$ a generic point in either one of the 1-parameter curves. The same argument given in the proof of Proposition 4.1 shows that these curves are rigid in $\mathcal{N}_{7,3}$.

(c) We also determine all the Lie algebras $\mathfrak{g} \in \mathcal{N}_{7,3}$ that are not in the 1parameter curves and have $\operatorname{dim} H_{3-n i l}^{2}(\mathfrak{g}, \mathfrak{g})=1$. For these Lie algebras we explicitly show a non-trivial deformation in $\mathcal{N}_{7,3}$ (showing that they are not rigid).

(d) As a byproduct, we point out a possible error in in Proposition 3.7 of GR (see item (3) below).

As a consequence of this, it is very likely that the four Lie algebras obtained in (a) are exactly the rigid points in $\mathcal{N}_{7,3}$. In order to complete the proof of this, one should consider the rigidity of all Lie algebras $\mathfrak{g} \in \mathcal{N}_{7,3}$ such that $\operatorname{dim} H_{3-n i l}^{2}(\mathfrak{g}, \mathfrak{g}) \geq 2$ (approximately 40 ). We think that all of them have non-trivial deformations in $\mathcal{N}_{7,3}$.

We will follow the classification of the 7-dimensional nilpotent Lie algebras over $\mathbb{R}$ given by Gong in $\mathrm{Go}$, and the one given by Seeley in $\mathrm{Se} 2$. The classification of Gong corrects some errors in the list given by Seeley. A more recent classification is given by Magnin in [Ma (see also [Ca]) but we will follow the classification of [Go] and Se2] since these authors list the Lie algebras by their upper central series (in $\mathrm{Ma}$ ] the Lie algebras are listed by rank). The list of 3-step nilpotent Lie algebras of dimension 7 in [Go] has 52 isolated real Lie algebras and two 1-parameter families of pairwise non-isomorphic nilpotent Lie algebras.

Rigid points and curves in $\mathcal{N}_{7,3}$. There are four 3-step nilpotent Lie algebras $\mathfrak{g}$ with $H_{3-\text { nil }}^{2}(\mathfrak{g}, \mathfrak{g})=0$ and thus they are rigid in $\mathcal{N}_{7,3}$. They are

$$
\mathfrak{g}_{137 B}, \quad \mathfrak{g}_{137 B_{1}}, \quad \mathfrak{g}_{247 H}, \quad \mathfrak{g}_{247 H_{1}}
$$

$\left(\mathfrak{g}_{137 B} \simeq \mathfrak{g}_{137 B_{1}}\right.$ and $\mathfrak{g}_{247 H} \simeq \mathfrak{g}_{247 H_{1}}$ over $\left.\mathbb{C}[\mathrm{Go}]\right)$ and the dimension of their orbits are, respectively, $36,36,38,38$.

The two 1-parameter families in $\mathcal{N}_{7,3}$ are

$$
\mathfrak{g}_{147 E}(t) \quad \text { and } \quad \mathfrak{g}_{147 E_{1}}(t), \quad \text { with } t>1
$$

(over $\mathbb{C}$, if $t=\cosh (\theta)>1$ then $\mathfrak{g}_{147 E_{1}}(t)$ is isomorphic to $\mathfrak{g}_{147 E}\left(t^{\prime}\right)$ with $\left.t^{\prime}=-\frac{(1-i \sinh (\theta))^{2}}{\cosh ^{2}(\theta)} \in \mathbb{C}\right)$. It turns out that, if

$$
\mathfrak{g}(t) \text { is either } \begin{cases}\mathfrak{g}_{147 E_{1}}(t) & \text { with } t>1, \text { or } \\ \mathfrak{g}_{147 E}(t) & \text { with } t>1, t \neq 2,\end{cases}
$$

then $\operatorname{dim} H_{3-n i l}^{2}(\mathfrak{g}(t), \mathfrak{g}(t))=1$ (and $\operatorname{dim} H_{3-n i l}^{2}\left(\mathfrak{g}_{147 E}(2), \mathfrak{g}_{147 E}(2)\right)=3$ ), and the non-zero cohomology class corresponds to the tangent vector of 
$\mathfrak{g}(t)$. Therefore, the same argument given in the proof of Proposition 4.1 . proves that

$$
\left\{\mathfrak{g}_{147 E}(t): 1<t<2\right\} \quad\left\{\mathfrak{g}_{147 E}(t): 2<t\right\} \quad\left\{\mathfrak{g}_{147 E_{1}}(t): 1<t\right\}
$$

are rigid curves in $\mathcal{N}_{7,3}$.

Non-trivial deformations of all $\mathfrak{g} \in \mathcal{N}_{7,3}$ with $\operatorname{dim} H_{3-\text { nil }}^{2}(\mathfrak{g}, \mathfrak{g})=1$. There are six other 3 -step nilpotent Lie algebras $\mathfrak{g}$ of dimension 7 in Go] (not members of the previous curves) such that $\operatorname{dim} H_{3-n i l}^{2}(\mathfrak{g}, \mathfrak{g})=1$. They are,

$$
\mathfrak{g}_{247 G}, \quad \mathfrak{g}_{247 K}, \quad \mathfrak{g}_{147 D}, \quad \mathfrak{g}_{137 A}, \quad \mathfrak{g}_{137 D}, \quad \mathfrak{g}_{137 A_{1}}
$$

None of them is rigid, in fact we claim that

$$
\begin{aligned}
\mathfrak{g}_{247 H} & \rightarrow \mathfrak{g}_{247 K} & & (\text { see item 4) } \\
& \searrow \mathfrak{g}_{247 G} & & (\text { see item 3) } \\
\mathfrak{g}_{137 B} & \rightarrow \mathfrak{g}_{137 A} & & \text { (see item 1) } \\
& \searrow \mathfrak{g}_{137 D} & & \text { (see item 1) } \\
\mathfrak{g}_{137 B_{1}} & \rightarrow \mathfrak{g}_{137 A_{1}} & & \text { (see item 1) } \\
\mathfrak{g}_{147 E_{1}}(t) & \rightarrow \mathfrak{g}_{147 D}, \text { as } t \rightarrow 1 . & & \text { (see item [2) }
\end{aligned}
$$

Next we prove these statements and point out an error that occur in GR].

(1) It is not difficult to see that $\mathfrak{g}_{137 B} \rightarrow \mathfrak{g}_{137 A}$ and $\mathfrak{g}_{137 B_{1}} \rightarrow \mathfrak{g}_{137 A_{1}}$ since

$$
\begin{aligned}
\mathfrak{g}_{137 A}: a b & =e, a e=g, c d=f, c f=g ; \\
\mathfrak{g}_{137 B}: a b & =e, a e=g, c d=f, c f=g, b d=g ; \\
\mathfrak{g}_{137 A_{1}}: a c & =e, a d=f, a e=g, b c=-f, b d=e, b f=g ; \\
\mathfrak{g}_{137 B_{1}}: a c & =e, a d=f, a e=g, b c=-f, b d=e, b f=g, c d=g .
\end{aligned}
$$

In addition, let

$\mathfrak{g}(t): a b=e, a d=f, a f=g, b c=f, b d=g, c d=-t^{2} e, c e=-g$.

If we rewrite the structure table of $\mathfrak{g}(t)$ in the basis

$\left\{t a+c, 2 t(t b-d),-t a+c,-2 t(t b+d), 4 t^{2}(t e-f), 4 t^{2}(t e+f),-8 t^{3} g\right\}$

we obtain the structure table of $\mathfrak{g}_{137 B}$. Since $\mathfrak{g}(0) \simeq \mathfrak{g}_{137 D}$ (same structure table), it follows that $\mathfrak{g}_{137 B} \rightarrow \mathfrak{g}_{137 D}$.

(2) The structure table of $\mathfrak{g}_{147 E_{1}}(t)$ is

$$
\begin{aligned}
\mathfrak{g}_{147 E_{1}}(t): a b=d, a c=-f, a f=-t g, & \\
& b c=e, b e=t g, b f=2 g, c d=-2 g .
\end{aligned}
$$

If we set $t=1$ and rewrite this table in the basis

$$
\{-a, a+b, c,-d, e-f,-f,-g\}
$$

we obtain the structure table of $\mathfrak{g}_{147 D}$

$\mathfrak{g}_{147 D}: a b=d, a c=-f, a e=g, a f=g, b c=e, b f=g, c d=-2 g$. 
(3) The structure table of $\mathfrak{g}_{247 G}$ is that of $\mathfrak{g}(0)$ where,

$$
\begin{aligned}
\mathfrak{g}(t): a b & =d, a c=e, a d=\left(1+\frac{t^{3}}{2}\right) f+\frac{t^{3}}{2} g, a e=\left(1-\frac{t^{3}}{2}\right) f-\frac{t^{3}}{2} g, \\
b d & =f, b e=g, c d=g, c e=f .
\end{aligned}
$$

On the other hand, if we rewrite the structure table of

$$
\mathfrak{g}_{247 H}: a b=d, a c=e, a d=f, b d=f, b e=g, c d=g, c e=f
$$

in the basis

$$
\begin{array}{r}
\left\{2 t^{2} a+\left(\frac{1}{2}-t^{2}\right) b+\frac{1}{2} c, \frac{1}{2}(1+t) b+\frac{1}{2}(1-t) c, \frac{1}{2}(1-t) b+\frac{1}{2}(1+t) c,\right. \\
t^{2}(1+t) d+t^{2}(1-t) e, t^{2}(1-t) d+t^{2}(1+t) e, \\
\left.t^{2}\left(1+t^{2}\right) f+t^{2}\left(1-t^{2}\right) g, t^{2}\left(1-t^{2}\right) f+t^{2}\left(1+t^{2}\right) g\right\},
\end{array}
$$

it coincides with the table of $\mathfrak{g}(t)$. This shows that $\mathfrak{g}(t) \simeq \mathfrak{g}_{247 H}$ for $t \neq 0$.

(4) In GR it is claimed that $\mathfrak{g}_{247 K}$ is rigid in $\mathcal{N}_{7,3}$. Next, we will show that this is not the case. The Lie algebra claimed to be rigid in [GR] is the following,

$\mathfrak{g}: a b=c, a c=d, a e=f, a f=g, b c=d, b e=f, c e=g$, ef $=d$.

Now, via the change of basis given by $\{b,-a+b, e, c, f, d,-g\}$ the structure table of $\mathfrak{g}$ becomes the same as that of $g_{247 K}$,

$$
\mathfrak{g}_{247 K}: a b=d, a c=e, a d=f, b e=g, c d=g, c e=f .
$$

Moreover, $\mathfrak{g}_{247 K}$ is $\mathfrak{g}(0)$ for the curve of Lie algebras,

$$
\mathfrak{g}(t): a b=d, a c=e, a d=f, b c=t^{2} e, b e=g, c d=g, c e=f .
$$

On the other hand, the structure table of $\mathfrak{g}_{247 H}$ (which is rigid) is

$$
\mathfrak{g}_{247 H}: a b=d, a c=e, a d=f, b e=g, b d=f, c d=g, c e=f
$$

and with respect to the basis given by

$$
\left\{-i a,-i t^{2}(a-b), t c, t^{2} d,-i t e,-i t^{2} f, t^{3} g\right\},
$$

yields the structure table of $\mathfrak{g}(t)$, showing that $\mathfrak{g}(t) \simeq \mathfrak{g}_{247 H}$ over $\mathbb{C}$ for all $t \neq 0$. Since $\mathfrak{g}_{247 H} \nsucceq \mathfrak{g}_{247 K}$ we obtain that the Lie algebra $\mathfrak{g}_{247 K}$ is not rigid. This also shows that $\mathfrak{g}_{247 H} \rightarrow \mathfrak{g}_{247 K}$ as we wanted to prove.

\section{REFERENCES}

[Bu] D. Burde, Degenerations of 7-dimensional nilpotent lie algebras, Comm. in Algebra, 33(2005), 1259-1277. DOI: 10.1081/AGB-200053956 4.3

[Ca] R. Carles, Weight systems for complex nilpotent Lie algebras and application to the varieties of Lie algebras, Publ. Univ. Poitiers, 96 (1996). 4.34 .4

[CSS] M. Crainic, F. Schätz and I. Struchiner, A survey on stability and rigidity results for Lie algebras, Indagationes Mathematicae, 25 (2014), 957-976. 1.11 .2

[CdGS] S. Cicalò, W. de Graaf, C. Schneider, Six-dimensional nilpotent Lie algebras, Linear Algebra and its Applications, 436 (2012), 163-189 3.1. 4.2

[dG] W. de Graaf, Classification of 6-dimensional nilpotent Lie algebras over fields of characteristic not 2, J. of Algebra, Vol. 309, 640-653 4.1

[Go] M. P. Gong, Classification of nilpotent Lie algebras of dimension 7 (over algebraically closed fields and R, Ph.D. thesis, University of Waterloo, Waterloo, Canada, 1998. 4.4 
[GA] M. Goze and J. M. Ancochea Bermudez, On the varieties of nilpotent Lie algebras of dimension 7 and 8, J. of Pure and Applied Algebra 77 (1992) 131-140. 4.3

[GR] M. Goze and E. Remm, k-step nilpotent Lie algebras, Georgian Math. J. Vol 22, (2015) 219-234. 1.2 囵 4.4, 田

[GK] M. Goze and Y. Khakimdjanov, Nilpotent Lie algebras, Mathematics and its Applications, 361, Kluwer Academic Publishers Group, Dordrecht, (1996).Manuscripta Math. 84 (1994), 115-224. 1.1

[GO] F. Grunewald, J. O'Halloran, Varieties of nilpotent Lie algebras of dimension less than six, J. Algebra 112 (1988), 31-325.

[H] R. S. Hamilton, The inverse function theorem of Nash and Moser, Bull. Amer. Math. Soc., 7 (1982), 65-222. 1.1

[Ma] L. Magnin, Adjoint and Trivial Cohomology Tables for Indecomposable Nilpotent Lie Algebras of Dimension $\leq 7$ over $\mathbb{C}$, eBook, 2nd Corrected Edition 2007, http://math.u-bourgogne.fr/topology/magnin/public_html/Magnin2.ps 4.3 4.3. 4.4

[Mo] J. Moser, A rapidly convergent iteration method and non-linear differential equations, Ann. Scuola Norm. Sup. Pisa (3) 20 (1966), 499-535. 1.1

[Na] J. Nash, The embedding problem for Riemannian manifolds, Ann. of Math. (2) 63 (1956), 20-63. 1.1

[NR] A. Nijenhuis and R. W. Richardson, Jr., Cohomology and deformations in graded Lie algebras, Bull. Amer. Math. Soc., 73 (1967), 175-179. 1.1

[R] R. W. Richardson, Jr., On the rigidity of semi-direct products of Lie algebras, Pacific Journal of Mathematics, 22, No. 2, (1967), 339-344. 1.2 4.1

[Se1] C. Seeley, Degenerations of 6-dimensional nilpotent Lie algebras over $\mathbb{C}$, Comm. in Algebra 18 (1990), 3493-3505. 3.1, 4.2

[Se2] C. Seeley, 7-dimensional nilpotent Lie algebras, Transactions of the American Mathematical Society, vol. 335 (1993), 479-496. 4.3, 4.4

[S] JP. Serre, Lie Algebras and Lie Groups: 1964 Lectures given at Harvard University, Lecture Notes in Mathematics, Springer, 2nd Edition. 1.1

[V] M. Vergne, Cohomologie des algèbres de Lie nilpotentes. Application à l'étude de la variété des algèbres de Lie nilpotentes, Bull. Soc. Math. France 98(1970) 81-116. 2

CiEm-COniCET, FAMAF-Universidad Nacional de Córdoba

E-mail address: brega@famaf.unc.edu.ar

CIEM-CONiCET, FAMAF-Universidad Nacional de CóRdoba

E-mail address: cagliero@famaf.unc.edu.ar

CIEM-CONICET, FAMAF-Universidad Nacional de Córdoba

E-mail address: aeco03@yahoo.com 